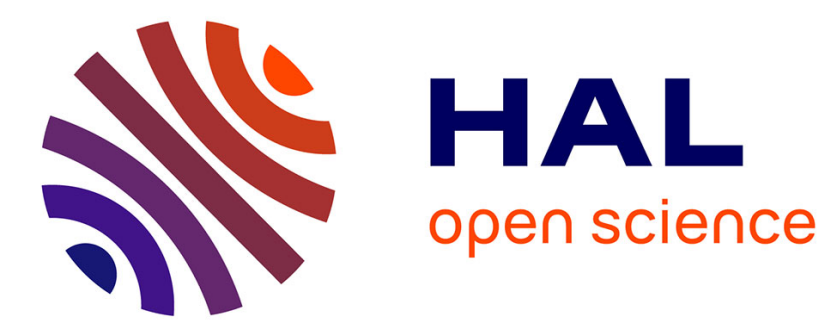

\title{
Self-defocusing in ultrasonic hyperthermia: Experiment and simulation
}

\author{
Claire Le Floch, Mickaël Tanter, Mathias Fink
}

\section{To cite this version:}

Claire Le Floch, Mickaël Tanter, Mathias Fink. Self-defocusing in ultrasonic hyperthermia: Experiment and simulation. Applied Physics Letters, 1999, 74 (20), pp.3062-3064. 10.1063/1.124065 . hal-02481857

\section{HAL Id: hal-02481857 https://hal.science/hal-02481857}

Submitted on 17 Feb 2020

HAL is a multi-disciplinary open access archive for the deposit and dissemination of scientific research documents, whether they are published or not. The documents may come from teaching and research institutions in France or abroad, or from public or private research centers.
L'archive ouverte pluridisciplinaire HAL, est destinée au dépôt et à la diffusion de documents scientifiques de niveau recherche, publiés ou non, émanant des établissements d'enseignement et de recherche français ou étrangers, des laboratoires publics ou privés. 


\section{Self-defocusing in ultrasonic hyperthermia: Experiment and simulation}

Cite as: Appl. Phys. Lett. 74, 3062 (1999); https://doi.org/10.1063/1.124065

Submitted: 03 November 1998 . Accepted: 17 March 1999. Published Online: 11 May 1999

Claire Le Floch, Mickaël Tanter, and Mathias Fink

\section{ARTICLES YOU MAY BE INTERESTED IN}

Focusing and steering through absorbing and aberrating layers: Application to ultrasonic propagation through the skull

The Journal of the Acoustical Society of America 103, 2403 (1998); https://

doi.org/10.1121/1.422759

Simulations of the thermo-acoustic lens effect during focused ultrasound surgery

The Journal of the Acoustical Society of America 109, 2245 (2001); https://

doi.org/10.1121/1.1360239

\section{Echo-to-reverberation enhancement using a time reversal mirror}

The Journal of the Acoustical Society of America 115, 1525 (2004); https://

doi.org/10.1121/1.1649737

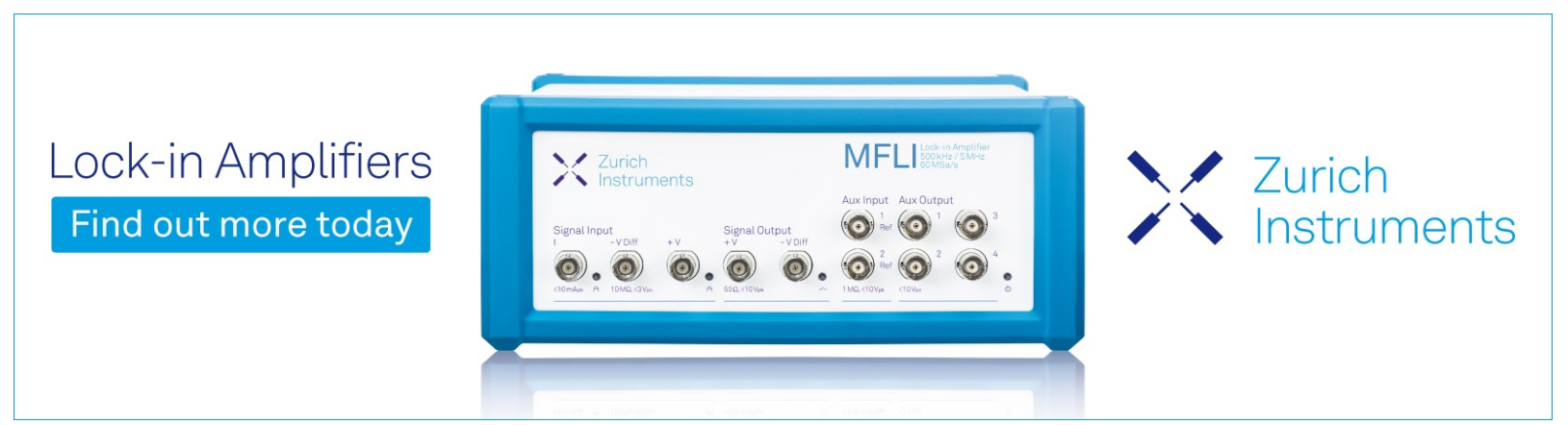




\title{
Self-defocusing in ultrasonic hyperthermia: Experiment and simulation
}

\author{
Claire Le Floch ${ }^{\text {a) }}$ \\ Laboratoires d'Electronique Philips S.A.S., 22 av. Descartes, 94450 Limeil Brevannes, France \\ Mickaël Tanter and Mathias Fink \\ Laboratoire Ondes et Acoustique, Ecole Supérieure de Physique et Chimie Industrielles de la Ville \\ de Paris, Université Paris VII, URA CNRS 1503, 10 rue Vauquelin, 75005 Paris, France
}

(Received 3 November 1998; accepted for publication 17 March 1999)

\begin{abstract}
During hyperthermia treatments with high intensity focused ultrasounds, the temperature elevation locally induces a change of sound speed which acts itself as an aberrator for the ultrasonic beam. Such a thermal lens effect may become noticeable as time increases and leads to significant effects like the self-defocusing of the beam. This letter presents an experimental evidence of this effect, and a finite difference simulation of the coupled wave and heat equations is presented that demonstrates similar phenomenon. () 1999 American Institute of Physics. [S0003-6951(99)02620-0]
\end{abstract}

Hyperthermia has become a wide field of investigations in the ultrasound community for the last few years. Such an ultrasonic noninvasive therapy requires both a precise control of the temperature elevation during the treatment, and a precise location of the focus. This focusing of high intensity ultrasonic beams is usually predicted by assuming the sound speed in the medium to be a constant $c(\mathbf{r}, t)=c_{0}$ ( $\mathbf{r}$ is the position vector and $t$ is the time). However, during hyperthermia, spatial distribution of temperature $T(\mathbf{r})$ leads to spatial variations of the sound speed $c(\mathbf{r}, t)=c[\mathbf{r}, T(\mathbf{r}, t)]$. This nonlinear process in the sound field may become noticeable as time increases and significant effects like self-defocusing of the sound beam ${ }^{1,2}$ occur. Self-defocusing of the acoustic beam in biological media generates important displacements of the thermal focus along the beam direction. As they involve dramatic damages during hyperthermia treatments, these displacements of the thermal focus have to be studied and understood more precisely. First, we present experimental results that show the self-defocusing effect in a fat mimicking medium where the sound speed decreases with temperature. This experiment is then correlated and discussed with a finite differences numerical solution of the coupled wave and heat transfer equations.

Recently, an ultrasonic technique has been proposed to noninvasively control temperature distribution during hyperthermia treatments. The scattering medium is scanned during the treatment. Changes of ultrasonic velocity with temperature induce apparent displacements in the ultrasonic speckle pattern of the medium. Cross-correlation measurements of these local displacements lead to an estimation of the temperature. Knowing the sound speed in the medium $c_{0}$ at an initial temperature $T_{0}$, the temperature change $\delta T$ is estimated from the time shift $\delta t$ in the echo signal by ${ }^{3,4}$

$$
\delta \mathbf{T} \approx \frac{c_{0}}{2} \frac{1}{\alpha-\left.\frac{1}{c_{0}} \frac{\partial \mathbf{c}}{\partial \mathbf{T}}\right|_{T_{0}}} \frac{\partial}{\partial z}(\delta t),
$$

where $\alpha$ is the linear coefficient of thermal expansion and $z$ is the imaging beam direction. Following that process, temperature distributions have been estimated in a rubber phantom. The experimental setup used is presented in Fig. 1. A $45 \mathrm{~mm}$ diameter spherical annular array emits high intensity focused ultrasounds in the medium. Its focus is located at $130 \mathrm{~mm}$ and is $3 \mathrm{~mm}$ wide and $40 \mathrm{~mm}$ long. The phantom is a rectangular rubber sample $(8 \mathrm{~cm} \times 4 \mathrm{~cm} \times 6 \mathrm{~cm})$, consisting of neoprene powder randomly embedded in a rubber material (absorption coefficient: $\mu=27 \mathrm{~Np} \mathrm{~m}^{-1}$ ). The sound speed in the phantom has been measured, and decreases when temperature increases, $(\partial \mathbf{c} / \partial \mathbf{T})=-3.68 \mathrm{~m} \mathrm{~s}^{-1}{ }^{\circ} \mathrm{C}^{-1}$, which is very close to fatty media values. The linear coefficient of thermal expansion is $\alpha=3.2 \times 10^{-4}{ }^{\circ} \mathrm{C}^{-1}$. In a plane $(X, Y)$ orthogonal to the heating beam direction, a 128 elements, 5 $\mathrm{MHz}$, electronically focused, linear array is used to monitor temperature. Temperature estimations in this material are presented in Fig. 2 for a $420 \mathrm{~W} / \mathrm{cm}^{2}$ acoustic power at the focus. The gray level intensity represents the temperature elevation in the medium, from 0 (black) up to $15^{\circ} \mathrm{C}$ (white).

Temperature elevation at the focus along the $x$ direction is also presented in Fig. 3. The temperature normally increases in the first $10 \mathrm{~s}$, with a Gaussian-like shape. But after a $12 \mathrm{~s}$ insonification, the temperature does not increase any longer, and an annular pattern appears. This experiment is an example of the self-defocusing of the ultrasonic beam. The heat deposition in the medium constitutes a localized aberrator for the beam itself, due to the local change of sound speed in the medium. The sound speed in the phantom de-

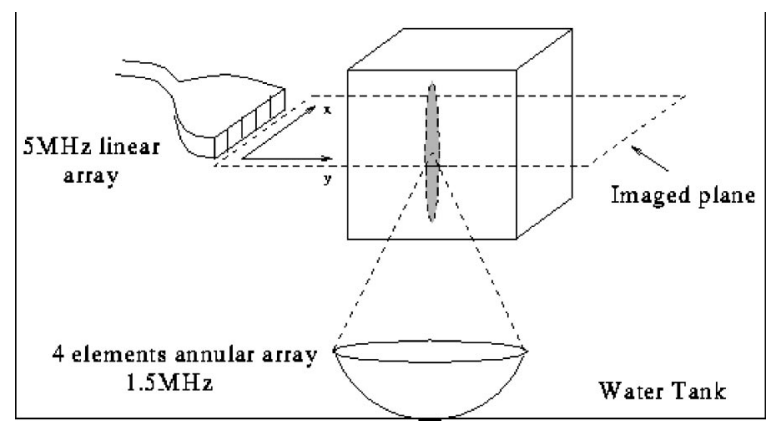

FIG. 1. Experimental setup used for temperature measurements.

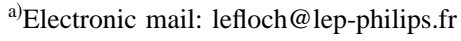




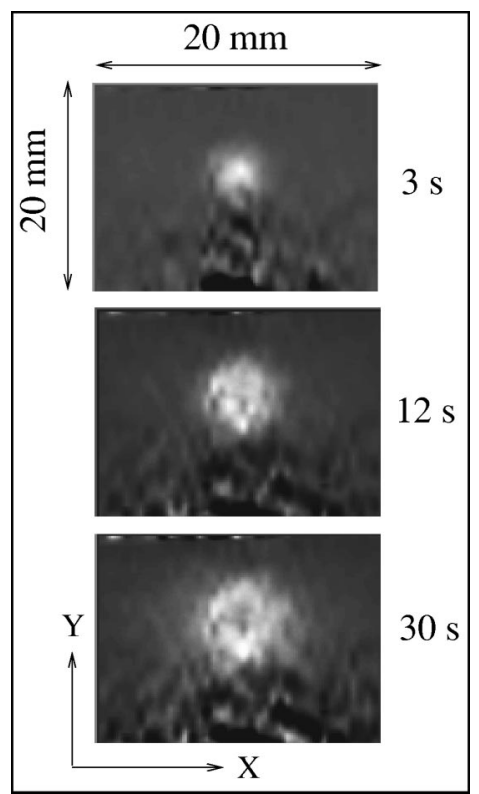

FIG. 2. Temperature distributions estimated in the rubber phantom after 3, 12 , and 30 s heating.

creases with temperature leading to a nearer focal point. The focus has moved out of the plane used for the temperature estimation.

To analyze precisely the phenomenon, a finite differences numerical simulation has been developed and is presented in the next section.

In order to explain this ultrasound self-defocusing, a nonlinear formulation, which allows for a change in the sound field due the acoustic heating is introduced by coupling the wave and heat equations. In particular, by assuming the acoustic streaming effect negligible compared to the acoustic heating, we obtain the coupled equations:

$$
\begin{aligned}
& \rho\left(1+\tau \frac{\partial}{\partial t}\right) \cdot \nabla\left[\frac{1}{\rho} \nabla p(\mathbf{r}, t)\right]-\frac{1}{c^{2}(\mathbf{r}, T)} \frac{\partial^{2} p(\mathbf{r}, t)}{\partial t^{2}}=S(\mathbf{r}, t) \\
& \rho c_{p} \frac{\partial T(\mathbf{r}, t)}{\partial t}-\frac{\kappa}{T_{0}} \Delta T(\mathbf{r}, t)=\mu \frac{p^{2}(\mathbf{r}, t)}{Z} \\
& c(\mathbf{r}, T)=f[T(\mathbf{r}, t)]
\end{aligned}
$$

where $p(\mathbf{r}, t)$ is the pressure field generated in the medium by the acoustic sources $S(\mathbf{r}, t), T(\mathbf{r}, t)$ is the temperature distribution, $c(\mathbf{r}, T)$ the sound speed in the medium, $\tau$ is a constant relaxation time that characterized the absorption in

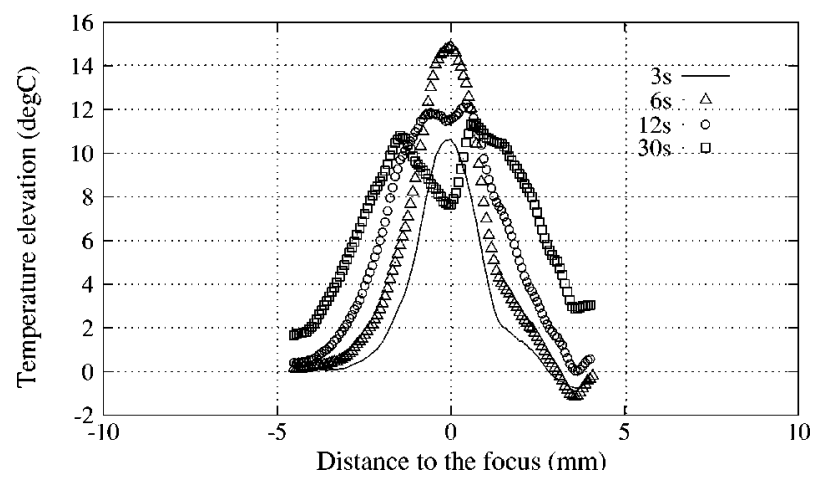

FIG. 3. Temperature elevation at the focus along the $x$ direction.

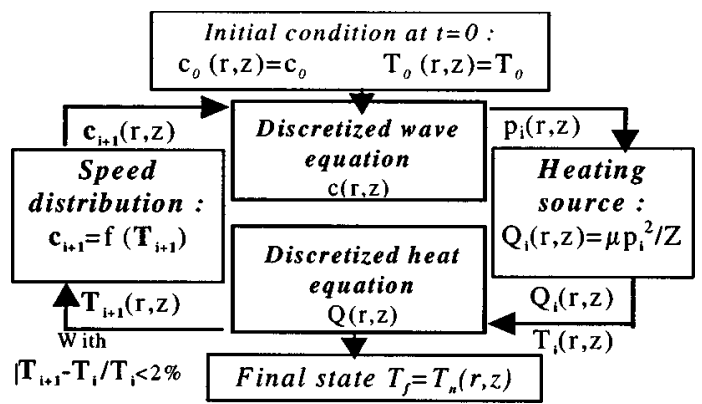

FIG. 4. Finite differences simulation of the coupled heat and wave equations.

the medium and $\mu$ the absorption coefficient. $\rho$ is the medium density, $c_{p}$ its specific heat, and $k$ the diffusion coefficient. The pure nonlinear sound-sound interaction is neglected.

To solve these coupled equations, the same spatial twodimensional grid in cylindrical coordinates $(r, z)$ is used to discretize both Eqs. (1) and (2). The grid consists of 410 $\times 1600$ points. The spatial steps $\Delta r$ and $\Delta z$ is equal to 0.1 $\mathrm{mm}$. A cylindrical array of sources located at the position $z$ $=0 \mathrm{~mm}$ generates a monochromatic beam $(f=1 \mathrm{MHz})$ focused in water at the position $z=130 \mathrm{~mm}$ along the axial direction. The ratio aperture/focal corresponds to the ratio used in the experiment. The time step for Eqs. (1) and (2) is respectively equal to $0.036 \mu$ s and $0.4 \mathrm{~ms}$. High order absorbing boundary conditions are used for the wave equation and $T$ is assumed to remain constant on the boundaries for the heat equation. Initial conditions are: $T(r, t \leqslant 0)=T_{0}$ and $S(r, t \leqslant 0)=0$.

We assume that variations of sound speed is a slowly varying function in time and space compared to the pressure field. In that case, the acoustic heating can be considered as a steady state temperature field with respect to the rapidly oscillating sound field. Then, the propagation and the heat equation can be treated separately:

(1) Equation (2) is initially solved for $0<t<t_{1}$ with an acoustic heating term deduced from the monochromatic distribution of sound field in the medium. The final time $t_{1}$ is reached when the relative changes in the spatial temperature distribution reach a $2 \%$ chosen threshold. This assumes that the acoustic field giving rise to the heating source is unchanged for $0<t<t_{1}$.

(2) The new spatial temperature distribution $T_{1}$ $=T\left(r, z, t=t_{1}\right)$ is now used to calculate the new sound speed distribution $c_{1}(r, z)=c\left(r, z, t=t_{1}\right)$ using Eq. (3).

(3) This new spatial sound speed inhomogeneity induces a change of the sound field according to Eq. (1). The wave equation can now be solved again to calculate the monochromatic acoustic field for $t_{1}<t<t_{2}$. This complete procedure is repeated step by step deducing the temperature field $T_{i+1}$ from $T_{i}$ on a time domain $0<t_{1}<\ldots<t_{i}<\ldots<t_{n}=t_{\text {final }}$, see Fig. 4.

All parameters are chosen to simulate the previous experiment in the rubber phantom. Figure 5 shows the simulated pressure field after a 3 and 30 s heating.

Due to attenuation along the acoustic path, the initial focus is $7 \mathrm{~mm}$ nearer $(z=123 \mathrm{~mm})$ than the transducer geometrical focus in water. The acoustic focusing is clearly dis- 


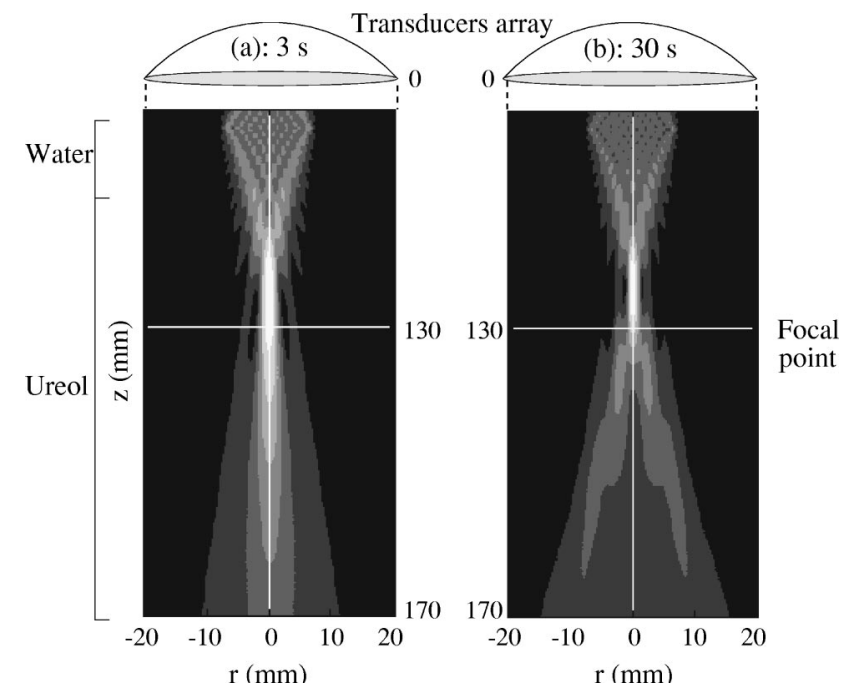

FIG. 5. Pressure field in the plane $(r, z)$ after (a) $3 \mathrm{~s}$ and (b) $30 \mathrm{~s}$ of monochromatic insonification.

torted during the heating. The heated area acts as a moving convergent lens for the ultrasonic beam, leading to a sharper focus, which moves $10 \mathrm{~mm}$ away from its initial position. The effect on the temperature distribution is shown in Fig. 6: a $3 \mathrm{~mm}$ displacement is observed along the axial direction.

It thus explains why the temperature elevation measured at the initial focus location is lower than expected. However, the two peaks observed in the experiment (Fig. 3) are not

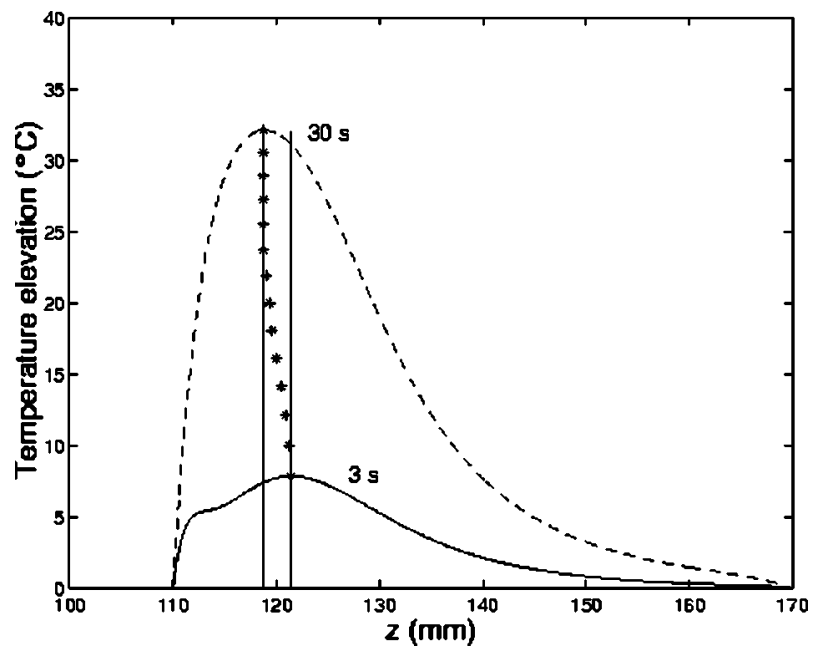

FIG. 6. Temperature elevation along the axial direction of the array after 3 (solid line) and $30 \mathrm{~s}$ (dashed line) of insonification.

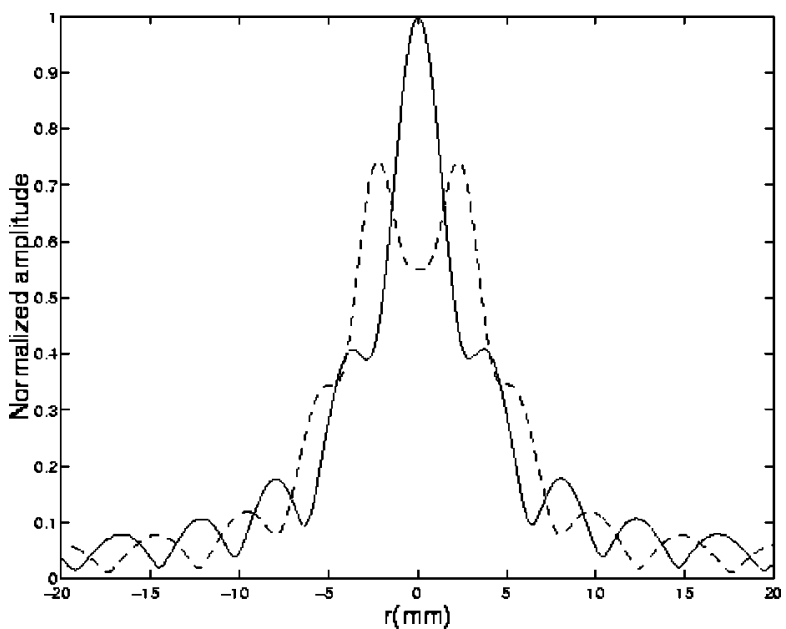

FIG. 7. Normalized pressure amplitude along the lateral direction of the array after a 3 (solid line) and a $30 \mathrm{~s}$ (dashed line) of insonification at $z$ $=130 \mathrm{~mm}$.

predicted by the simulation. Several hypothesis could be made to explain this difference:

(1) Figure 7 shows that the pressure field along the lateral direction after $30 \mathrm{~s}$, begins to be divided into two patterns just behind the focus $(30 \mathrm{~mm})$. This effect does not appear on the temperature profile because of diffusion phenomenon. The diffusion coefficient plays a very important part in the shape of the temperature profile, and may depend on temperature too. This potential dependence is not included in the simulation.

(2) The simulation does not take into account the dependence of the absorption coefficient on temperature, which may lead to very different heat deposit in the medium, and change the beam distortion.

In conclusion, it has been experimentally demonstrated that acoustic heating induces a significant self-defocusing effect of the ultrasonic beam during hyperthermia treatments. This self-defocusing is very sensitive to the emitted power and the axial size of the focal spot. To avoid dramatic damages, the problem of correction of such focal displacements has to be studied in detail in order to find the optimal treatment configuration (power and duration of the emission, focus shape, and if necessary adaptive focusing techniques).

${ }^{1}$ S. Makharov and M. Ochmann, Acustica 82, 579 (1996).

${ }^{2}$ V. Yu. Armeev, A. A. Karabutov, and O. A. Sapozhnikov, Sov. Phys. Acoust. 33, 109 (1987).

${ }^{3}$ R. Seip, P. VanBaren, C. A. Cain, and E. S. Ebbini, IEEE Trans. Ultrason. Ferroelectr. Freq. Control 43, 1063 (1996).

${ }^{4}$ C. Simon, P. VanBaren, and E. S. Ebbini, IEEE Ultrasonic Symp. 2, 1319 (1997). 